\title{
Papel do Endotélio na COVID-19 Grave
}

\author{
The Role of the Endothelium in Severe COVID-19
}

Simone Cristina Soares Brandão, ${ }^{\circledR}$ Emmanuelle Tenório Albuquerque Madruga Godoi, ${ }^{1}$ Júlia de Oliveira Xavier Ramos, ${ }^{1}$ Leila Maria Magalhães Pessoa de Melo, ${ }^{2}$ Luca Terracini Dompieri, ${ }^{10}$ Djair Falcão Brindeiro Filho, ${ }^{1}$ Emanuel Sávio Cavalcanti Sarinho ${ }^{1}$

Universidade Federal de Pernambuco, ${ }^{1}$ Recife, PE - Brasil

Serviço de Hematologia de São José dos Campos, ${ }^{2}$ São José dos Campos, SP - Brasil

\section{Introdução}

Estudos têm revelado uma relação significativa entre a gravidade da COVID-19 (COronaVIrus Disease 2019) e marcadores imunes. Sabe-se, por exemplo, que o endotélio participa ativamente da resposta imune e interage intimamente com o sistema de coagulação. ${ }^{1}$ Além disso, processos inflamatórios crônicos do endotélio estão envolvidos na fisiopatologia das doenças cardiovasculares (DCV) e metabólicas. ${ }^{2}$ Essas afecções podem impactar negativamente na evolução da COVID-19, e a resposta imune exacerbada do endotélio parece ser o fator determinante desse efeito. ${ }^{3}$

O coronavírus 2 da síndrome respiratória aguda grave (SARS-CoV-2) causa infecção por meio da ligação da proteína $\mathrm{S}$ ao receptor da enzima conversora de angiotensina 2 (ECA-2) na superfície da célula humana. ${ }^{3-5}$ Desse modo, é observada uma redução na disponibilidade dessa enzima, amplamente expressa em vários tecidos do corpo humano, notadamente em pulmões, coração e endotélio, com distúrbio na modulação do sistema renina-angiotensina-aldosterona (SRAA). ${ }^{6}$ Consequentemente, há um favorecimento da maior concentração de angiotensina 2 com uma série de ações deletérias ao organismo. Condições associadas à disfunção crônica do endotélio, como idade, hipertensão arterial sistêmica (HAS), DCV, diabetes melito e obesidade, são mais frequentes nos pacientes com COVID-19 grave (Figura 1). 2,7

Esse desequilíbrio no SRAA contribui para um estado próinflamatório, pró-oxidativo, com recrutamento macrofágico, excesso de citocinas circulantes, aumento na liberação de aldosterona, lesão tecidual e disfunção de múltiplos órgãos, característicos da forma grave da COVID-19.6,8,9 Todas essas alterações desencadeadas pelo SARS-CoV-2 podem prejudicar a função endotelial; por isso, comorbidades ligadas ao endotélio conferem maior gravidade à doença. Percebe-se, assim, na fisiopatogenia da COVID-19, uma inter-relação entre fatores pró-inflamatórios e pró-trombóticos, tornando-os importantes alvos terapêuticos. ${ }^{1,3,8-10}$

\section{Palavras-chave}

Doenças Cardiovasculares/fisiopatologia; COVID-19, Betacoronavirus; Endotélio; Imunidade; Aterosclerose; Trombose.

Correspondência: Simone Cristina Soares Brandão •

Universidade Federal de Pernambuco - Av. Prof. Moraes Rego, 1235.

CEP 50670-901, Cidade Universitária, Recife, PE - Brasil

E-mail: sbrandaonuclearufpe@gmail.com

Artigo recebido em 12/06/2020, revisado em 27/07/2020, aceito em 05/08/2020

DOI: https://doi.org/10.36660/abc.20200643

\section{Resposta do Endotélio à COVID-19}

O endotélio desempenha papel fundamental na resposta à infecção. Isso porque células endoteliais liberam substâncias solúveis, as quimiocinas, que atraem os leucócitos para o local infectado e produzem citocinas, que ativam a resposta inflamatória. Desse modo, pacientes com disfunção endotelial crônica apresentam alterações importantes no glicocálix, nas junções intercelulares e células endoteliais, o que resulta em maior adesão e extravasamento de leucócitos, induzindo um estado de hipercoagulabilidade e redução na ação fibrinolítica. A disfunção endotelial crônica contribui, então, para o desenvolvimento da COVID-19 grave. ${ }^{8,9}$

O endotélio é um órgão ativo, indispensável para a regulação do tônus e manutenção da homeostase vascular. ${ }^{1}$ Na COVID-19, o recrutamento de células imunes, seja pela agressão viral direta ao endotélio ou imunomediada, pode resultar em disfunção endotelial generalizada, associada à apoptose..$^{3,11-13}$ Estudos histológicos post-mortem revelaram um quadro de endotelite linfocítica em pulmões, coração, rins e fígado, bem como necrose celular e presença de microtrombos, que, nos pulmões, agravam a insuficiência respiratória. ${ }^{1,6,8,9,14,15}$

O endotélio já foi estudado em outras doenças virais, como no vírus da imunodeficiência humana (HIV) e na influenza. Assim como o HIV, o SARS-CoV-2 parece ter um efeito direto de agressão endotelial. ${ }^{13,16} \mathrm{Em}$ estudos de autópsias, foram encontradas evidências de agressão viral direta do SARSCoV-2 à célula endotelial e inflamação difusa. ${ }^{1,12}$ Ackermann et al. ${ }^{1}$ demonstraram uma quantidade de microtrombos nove vezes maior em pulmões de pessoas com COVID-19 do que naquelas com influenza. Nesses mesmos pulmões, a neoangiogênese também foi 2,7 vezes mais prevalente na COVID-19 do que na influenza.

A ideia de que estados inflamatórios crônicos subclínicos sejam responsáveis pela instalação de doenças ou pelo seu agravamento encontra-se bem estabelecida. A associação de células inflamatórias e seus respectivos produtos é bem reconhecida na fisiopatologia da aterosclerose, condição com grande repercussão no endotélio e nos componentes da síndrome metabólica (obesidade, diabetes melito e has). ${ }^{11,17}$

Embora as doenças cardiometabólicas possam iniciar na infância, é nas fases adulta e senil que ela é expressivamente mais prevalente. Na aterosclerose, assim como na COVID-19, existe predominância de resposta $T_{H} 1$, envolvendo o interferon-gama (IFN- $\gamma$ ), o fator de necrose tumoral alfa (TNF$\alpha$ ) e o beta (TNF- $\beta$ ), que amplificam a resposta inflamatória. $\mathrm{O}$ IFN- $\gamma$ é considerado uma das principais citocinas pró- 


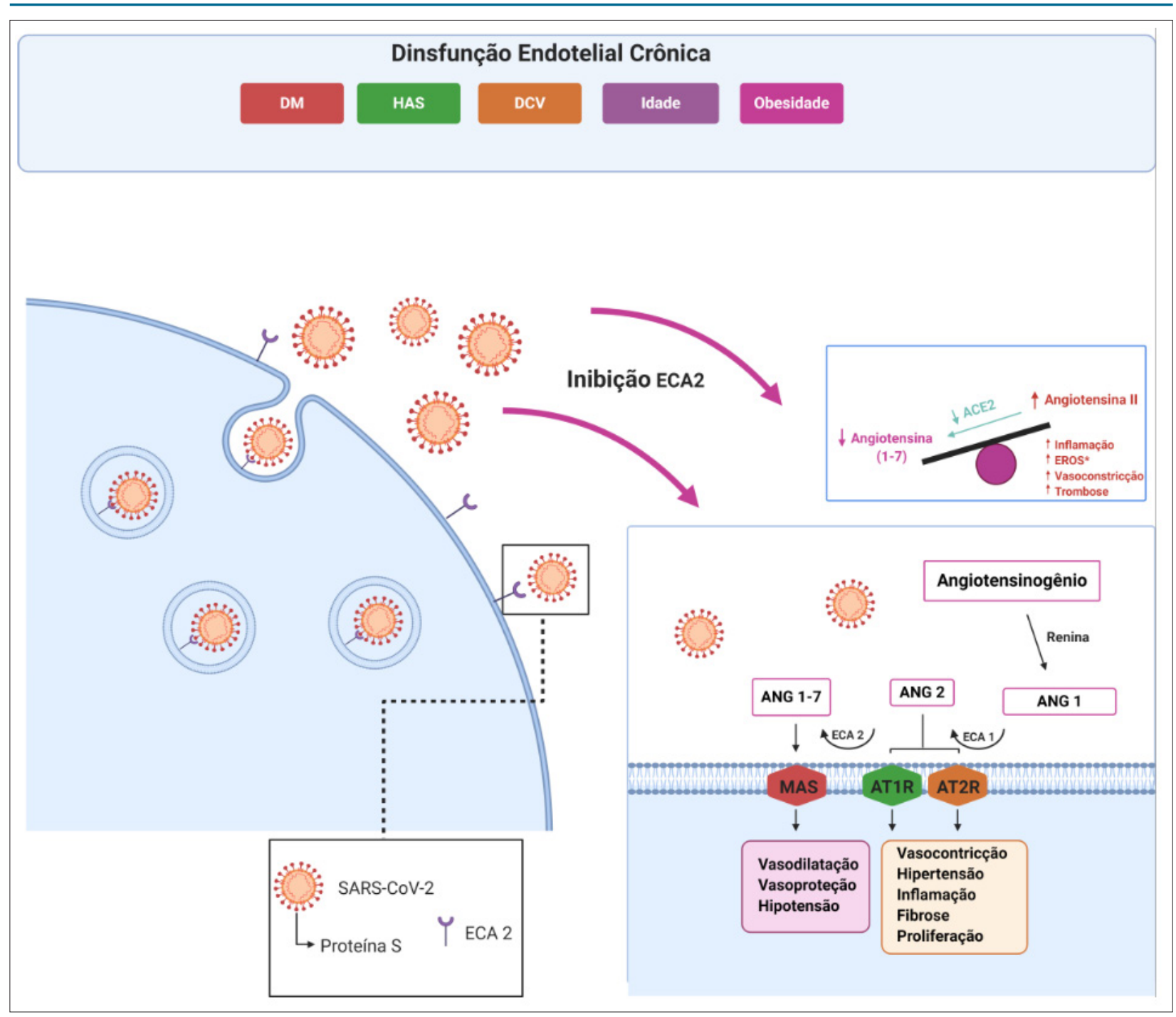

Figura 1 - Consequências da ligação do coronavírus 2 da síndrome respiratória aguda grave (SARS-CoV-2) com o receptor da enzima conversora de angiotensina 2 (ECA-2). A proteína $S$ do vírus se liga ao receptor da ECA-2 da célula humana, reduzindo sua atividade enzimática. A ECA-1 e a ECA-2 agem nas angiotensinas (ANG) 1 e 2, respectivamente. A hipofunção da ECA-2 leva a uma diminuição na concentração de ANG 1-7 e, consequentemente, a um aumento na quantidade de ANG 2, com efeitos deletérios a órgãos e tecidos. Comorbidades como diabetes melito, HAS, DCV, idade avançada e obesidade causam disfunção endotelial crônica, que é agravada pela desregulação do sistema renina-angiotensina-aldosterona ocasionada pelo SARS-CoV-2. DCV: doenças cardiovasculares; EROS: espécies reativas de oxigênio; HAS: hipertensão arterial sistêmica; AT1R: receptor 1 de ANG 2; AT2R: receptor 2 de ANG 2; R-MAS: receptor de angiotensina 1-7. Fonte: elaborada pelos autores. Criada com biorender.com

aterogênicas, pois ativa macrófagos e favorece a participação deles na resposta inflamatória. ${ }^{11,12}$ Torna-se evidente que uma amplificação do processo aterosclerótico ocorre a partir de uma resposta imune específica, com produção de citocinas da via $T_{H} 1$, como a interleucina-12 (IL-12) e o IFN- $\gamma .{ }^{11,12}$

Uma vez que o desequilíbrio no sistema imunológico está presente na fisiopatologia das DCV e da síndrome metabólica, as pessoas com essas doenças, até mesmo as mais jovens, com aterosclerose incipiente, estariam mais susceptíveis à forma grave da COVID-19, por já possuírem um "terreno" imune hiperativo e desregulado. 5,11,18

Outra explicação para as doenças cardiometabólicas serem fatores de risco para a forma grave da COVID-19 envolve os receptores de reconhecimento de patógenos toll-like-4 (TLR4), integrantes moleculares da imunidade inata. ${ }^{5,18}$ Já se sabe que os TLR4 participam da patogênese das DCV e metabólicas, como aterosclerose, diabetes e obesidade. Eles são expressos em diferentes tipos de células da placa aterosclerótica, e vários ligantes pró-aterogênicos podem ativá-los. Os TLR4 estão também envolvidos na lipotoxicidade e na disfunção de células betapancreáticas. A hiperexpressão dos TLR4 pode ser, inclusive, geneticamente codificada. ${ }^{5,18}$

$\mathrm{Na}$ imunopatologia da COVID-19 ocorre elevação, principalmente, da interleucina-6 (IL-6) e do TNF- $\alpha$. Essas citocinas são produtos de ativação do TLR4. Em um estudo por simulações computacionais, demonstrou-se que a proteína $S$ 
do SARS-CoV-2 é reconhecida pelos TLR4. ${ }^{5}$ Assim, indivíduos com maior expressão desses receptores, uma vez infectados pelo SARS-CoV-2, sofreriam maior ativação e liberação de IL-6 e TNF- $\alpha$, condição vista na forma grave da COVID-19.

Como já comentado, outro provável mecanismo responsável pela pior evolução da COVID-19 envolve o receptor da ECA-2. ${ }^{19}$ A redução da sua atividade pelo SARS-CoV-2 tem implicações nas DCV por potencializar a desregulação do SRAA e do sistema imune. ${ }^{6,20}$ Já existem evidências de que o uso de medicações que bloqueiam o SRAA, como inibidores de ECA-1 (IECA) e bloqueadores do receptor da angiotensina (BRA), não se relaciona com aumento de mortalidade pela COVID-19, podendo inclusive ser fator de proteção. ${ }^{19,21}$

\section{Alterações na Coagulação na COVID-19}

Estados inflamatórios exacerbados culminam em estase sanguínea, ativação plaquetária e disfunção endotelial, elevando as chances de episódios trombóticos venosos e arteriais. A coagulopatia na infecção grave por COVID-19 é semelhante à coagulopatia induzida pela sepse, caracterizada por coagulação intravascular disseminada e microangiopatia trombótica. Somado a isso, destaca-se que a hipoxemia, secundária à lesão pulmonar causada pela COVID-19, é fator de risco para trombose. ${ }^{8,9,17}$

O SARS-CoV-2 provoca a síndrome respiratória aguda grave (SARS), na qual ocorre acúmulo de fibrina insolúvel no espaço alveolar. Aventa-se que o fibrinogênio extravase do plasma por aumento da permeabilidade vascular e dano alveolar difuso, com eliminação incompleta devido a um estado de hipofibrinólise. Cronicamente, essa fibrina insolúvel contribui para fibrose pulmonar e seus desdobramentos negativos. $8,9,15,17$

As principais alterações na coagulação presentes na COVID-19 são: elevação do dímero-D, do fibrinogênio e do tempo de protrombina, e diminuição da fibrinólise. A contagem de plaquetas pode estar reduzida nos estágios mais avançados da doença, sendo fator preditivo de mortalidade. ${ }^{8,9,15,17} \mathrm{O}$ aumento do risco de trombose ocorre também nas artérias, e diferentes manifestações clínicas podem aparecer, como: acidente vascular encefálico, isquemia mesentérica, infarto agudo do miocárdio e oclusão arterial de membros inferiores, a depender do leito arterial acometido. ${ }^{22}$ Corroborando a hipótese de agressão vascular, alguns casos com características da síndrome do choque tóxico ou síndrome inflamatória multissistêmica pediátrica similar à doença de Kawasaki têm sido descritos e relacionados à COVID-19. ${ }^{20}$

\section{Estratégias Terapêuticas}

Considerando o exposto, destaca-se a importância do controle rigoroso dos fatores de risco cardiometabólicos. ${ }^{5}$ O objetivo é deixar o endotélio menos reativo e menos vulnerável à COVID-19. A otimização do tratamento medicamentoso com o uso de hipoglicemiantes, antihipertensivos, hipolipemiantes (principalmente as estatinas) e antiagregantes plaquetários (como o ácido acetilsalicílico) pode estabilizar o endotélio. ${ }^{5,20,23}$ Fármacos como IECA e BRA parecem fundamentais na redução do risco de desfechos graves pela COVID-19, uma vez que ajudam a equilibrar o SRAA. ${ }^{19}$ Em relação ao SARS-CoV-2, até o momento, não existe tratamento específico que seja comprovadamente eficaz no combate ao vírus. A estratégia terapêutica tem se baseado no reconhecimento precoce das complicações e no suporte otimizado para aliviar os sintomas (Figura 2).

Na fase hiperinflamatória da COVID-19, as medicações que inibam ou reduzam os efeitos das citocinas próinflamatórias são muito pertinentes e devem ser levadas em consideração. Os inibidores de IL-6, assim como os glicocorticoides, poderiam evitar ou amenizar a tempestade de citocinas. ${ }^{23}$ Novas medicações moduladoras da resposta inflamatória são fundamentais nessa fase para evitar a inflamação excessiva, que agride intensamente o endotélio e os diversos órgãos, podendo culminar com falência de múltiplos órgãos até a morte.

Em relação ao tromboembolismo venoso, os pacientes hospitalizados devem receber tromboprofilaxia farmacológica com heparina de baixo peso molecular ou fondaparinux (preferencialmente à heparina não fracionada), a menos que o risco de sangramento exceda o de trombose, quando então se faz a profilaxia mecânica. ${ }^{8,9,15,17} \mathrm{O}$ ajuste da dose da heparina de acordo com o índice de massa corpórea e com o clearance de creatinina é recomendado. 8,15 A heparinização plena é indicada em casos de forte suspeita clínica ou já confirmados de tromboembolismo venoso..$^{8,15}$

A heparina é uma medicação já largamente utilizada na medicina, pela ação anticoagulante e pelo efeito antiinflamatório. Entretanto, na COVID-19, além desses efeitos, estudos têm sugerido seu uso como modo de impedir a replicação viral. O SARS-CoV-2 se liga ao receptor da ECA-2 para penetrar na célula humana e se multiplicar. Acredita-se que, para essa ligação, o vírus também precisa se ligar ao heparan sulfato presente, dentre outros sítios, na membrana basal do endotélio. A utilização de heparina tem sido sugerida como estratégia de ligação ao heparan sulfato, impedindo a ligação do SARS-CoV-2 ao receptor da ECA-2 e diminuindo, assim, a replicação viral. ${ }^{24}$

\section{Conclusão}

Em resumo, ressalta-se que a função endotelial é fator fundamental na progressão dos estágios clínicos da COVID-19, pois a disfunção crônica do endotélio, que acontece nas doenças pré-existentes, favorece diretamente a evolução para a forma grave da doença. Assim, enquanto a vacina é aguardada, os alvos terapêuticos (Tabela 1) devem ser: controle das condições cardiovasculares, metabólicas e endoteliais da população de risco e nos infectados; e redução da replicação viral, da hiperinflamação e da hipercoagulabilidade.

\section{Contribuição dos Autores}

Concepção e desenho da pesquisa: Brandão SCS, Godoi ET, Sarinho ESC; Obtenção de dados: Brandão SCS, Godoi ET, Ramos JOX, Melo LMMP, Dompieri LT; Análise e interpretação dos dados: Brandão SCS, Ramos JOX, Melo LMMP, Dompieri LT, Sarinho ESC; Redação do manuscrito: Brandão SCS, Godoi 


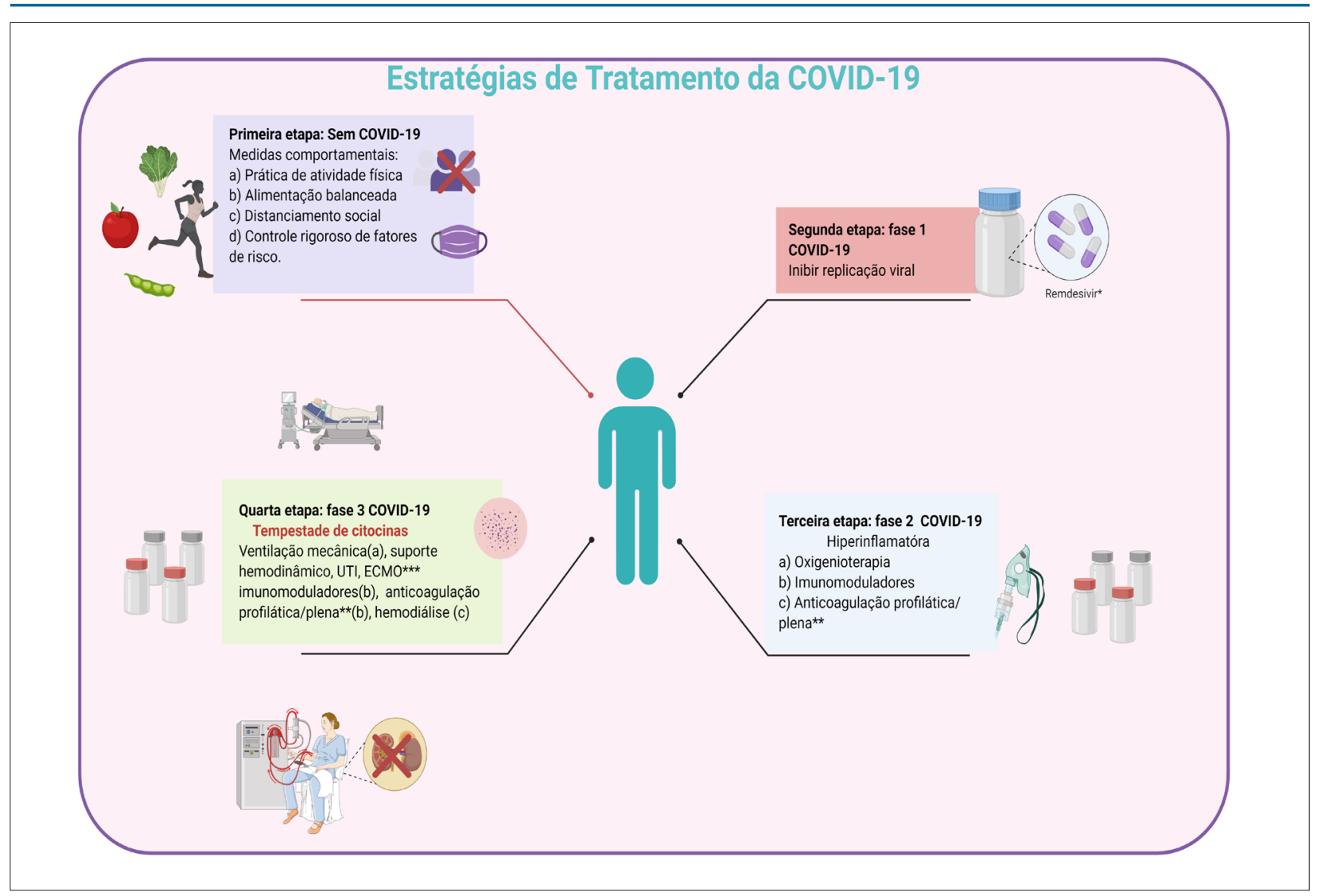

Figura 1 - Estratégias de tratamento para a prevenção da COVID-19 de acordo com as fases da doença. Fonte: elaborada pelos autores. Criada com biorender.com

ET, Ramos JOX, Melo LMMP, Sarinho ESC; Revisão crítica do manuscrito quanto ao conteúdo intelectual importante: Brandão SCS, Godoi ET, Brindeiro Filho DF, Sarinho ESC.

\section{Potencial Conflito de Interesses}

Declaro não haver conflito de interesses pertinentes.

\section{Fontes de Financiamento}

O presente estudo não teve fontes de financiamento externas.

\section{Vinculação Acadêmica}

Não há vinculação deste estudo a programas de pósgraduação. 


\begin{tabular}{|c|c|c|}
\hline Etapas & Potenciais alvos terapêuticos & Medicações \\
\hline \multirow{4}{*}{ Etapa 1: sem COVID-19 } & Anti-hipertensivos & $\begin{array}{l}\text { Inibidores da enzima conversora de angiotensina (ECA) e bloqueadores } \\
\text { dos receptores de angiotensina (BRA), principalmente }\end{array}$ \\
\hline & Estatinas & Sinvastatina, rosuvastatina, atorvastatina etc. \\
\hline & Antiplaquetários & Ácido acetilsalicílico \\
\hline & Vacinas & Múltiplas candidatas. Pesquisas em andamento \\
\hline \multirow{6}{*}{ Etapa 2: fase 1 da COVID-19 } & Entrada pelo receptor da ECA-2 & ECA-2 recombinante solúvel \\
\hline & TMPRSS2 protease S priming & Inibidor de protease (mesilato de camostato) \\
\hline & Endocitose do receptor & Cloroquina ou hidroxicloroquina* \\
\hline & RNA polimerase para replicação & Remdesivir, Favipiravir \\
\hline & Proteases virais & Lopinavir/Ritonavir* \\
\hline & Transporte nuclear pela importina & Ivermectina \\
\hline \multirow{7}{*}{$\begin{array}{l}\text { Etapas } 3 \text { e 4: } \\
\text { Fase } 2 \text { da COVID-19, hiperinflamatória } \\
\text { Fase } 3 \text { da COVID-19, "tempestade" de citocinas }\end{array}$} & Antivirais/anti-inflamatórios & $\begin{array}{l}\text { Plasma convalescente de pacientes com a COVID-19, Interferon tipo I, } \\
\text { imunoglobulinas, células troncomesenquimais }\end{array}$ \\
\hline & Ativação pelo excesso de interleucina-1 & Anakinra, canaquinumabe, colchicina \\
\hline & Tempestade de citocinas & $\begin{array}{l}\text { Tocilizumabe, sarilumabe, siltuximabe (inibidores de interleucina-6) ou } \\
\text { baricitinibe (inibidor da JAK), lenzilumab (inibidor do fator estimulante de } \\
\text { colônias de granulócito-macrófago) }\end{array}$ \\
\hline & Infecção bacteriana/inflamação & Azitromicina e outros antibióticos \\
\hline & Coagulopatia & Regime de anticoagulação plena ou profilática \\
\hline & Antivirais/anti-inflamatórios & Plasma convalescente \\
\hline & Estresse oxidativo & Vitamina C, deferoxamine \\
\hline
\end{tabular}

ECA 2: enzima conversora da angiotensina 2. TMPRSS2: transmembrana protease serina 2. Fonte: modificada da referência 10.

\section{Referências}

1. Ackermann M, Verleden SE, Kuehnel M, Haverich A, Welte T, Laenger F, et al. Pulmonary vascular endothelialitis, thrombosis, and angiogenesis in COVID-19. New Engl J Med. 2020;383(2):120-8.

2. Costa IBS S, Bittar CS, Rizk SI, Araújo Filho AE, Santos KAQ, Machado TIV, et al. The heart and COVID-19: what cardiologists need to know. Arq Bras Cardiol. 2020;114(5):805-16.

3. Bermejo-Martin JF, Almansa R, Torres A, González-Rivera M, Kelvin DJ.. COVID-19 as a cardiovascular disease: the potential role of chronic endothelial dysfunction. Cardiovasc Res. 2020 May 18:cvaa140.

4. Albini A, Di Guardo G, Noonan DM, Lombardo M. The SARS-CoV-2 receptor, ACE-2, is expressed on many different cell types: implications for ACE-inhibitor- and angiotensin II receptor blocker-based cardiovascular therapies. Intern Emerg Med. 2020;15(5):759-66

5. Choudhury A, Mukherjee S. In silico studies on the comparative characterization of the interactions of SARS-CoV-2 spike glycoprotein with ACE-2 receptor homologs and human TLRs. J Med Virol. 2020 May 8. [Epub ahead of print].

6. Brojakowska A, Narula J, Shimony R, Bander J. Clinical implications of SARS-CoV-2 interaction with renin angiotensin system. J Am Coll Cardiol. 2020;75(24):3085-95.

7. Chen T, Wu D, Chen H, Yan W, Yang D, Chen G, et al. Clinical characteristics of 113 deceased patients with coronavirus disease 2019: retrospective study. BMJ. 2020 March 26;368:m1091.
8. Klok FA, Kruip MJHA, Meer NJM, Arbours MS, Gommers DAMPJ, Kamt KM, et al. Incidence of thrombotic complications in critically ill ICU patients with COVID-19. Thromb Res. 2020;191:145-7.

9. Llitjos J, Leclerc M, Chochois C, Monsallier M, Ramakers M, Auvray M, etal. High incidence of venous thromboembolic events in anticoagulated severe COVID-19 patients. J Thromb Haemost. 2020;18(7):1743-6.

10. Liu PP, BletA, Smyth D, Li H. The science underlying COVID-19. Circulation. 2020;142(1):68-78.

11. Gimbrone Jr MA, García-Cardeña G. Endothelial cell dysfunction and the pathobiology of atherosclerosis. Circ Res. 2016;118(4):620-36.

12. Mehta P, McAuley DF, Brown M, Sanchez E, Tattersall RS, Manson JJ, et al. COVID-19: consider cytokine storm syndromes and immunosuppression. Lancet. 2020;395(10229):1033-4.

13. Gupta A, Madhavan MV, Landry DW. Extrapulmonary manifestations of COVID-19. Nat Med. 2020;26:1017-32.

14. Varga Z, Flammer AJ, Steiger P, Haberecker M, Andermatt R, Zinkernagel AS, et al. Endothelial cell infection and endotheliitis in COVID-19. Lancet. 2020;395(10234):1417-8.

15. Ramacciotti E, Macedo AS, Biagioni RB, Caffaro RA, Lopes RD, Guerra JC, et al. Evidence-based practical guidance for the antithrombotic management in patients with coronavirus disease (COVID-19) in 2020. Clin Appl Thromb Hemost. 2020;26:107602962093635. 
16. Godoi ETAM, Brandt CT, Lacerda HR, Godoi JTAM, Oliveira DC, Costa GFAS, et al. Intima-media thickness in the carotid and femoral arteries for detection of arteriosclerosis in human immunodeficiency virus-positive individuals. Arq Bras Cardiol. 2016; 108(1):3-11.

17. Petrilli CM, Jones SA, Yang J, Rajagopalan H, O’Donnel L, Chernyak Y, et al. Factors associated with hospital admission and critical illness among 5279 people with coronavirus disease 2019 in New York city: prospective cohort study. BMJ.2020;m1966.

18. Eguchi K, Manabe I. Toll-like receptor, lipotoxicity and chronic inflammation: the pathological link between obesity and cardiometabolic disease. J Atheroscler Thromb. 2014;21(7):629-39.

19. Zhang P, Zhu L, Cai J, Lei F, Qin JJ, Xie J, et al. Association of inpatient use of angiotensin-converting enzyme inhibitors and angiotensin II receptor blockers with mortality among patients with hypertension hospitalized with COVID-19. Circ Res. 2020;126(12):1671-81.
20. Zhao M, Wang M, Zhang J, Ye J, Xu Y, Wang Z, et al. Advances in the relationship between coronavirus infection and cardiovascular diseases. Biomed Pharmacother.2020 May 13;127:110230.

21. Guo T, Fan Y, Chen M, Wu X, Zhang L, He T, et al. Cardiovascular implications of fatal outcomes of patients with coronavirus disease 2019 (COVID-19). JAMA Cardiol.2020;5(7):1-8.

22. Divani AA, Andalib S, Di Napoli M, Lattanzi S, Hussain MS, Biller J, et al. Coronavirus disease 2019 and stroke: clinical manifestations and pathophysiological insights. J Stroke Cerebrovasc Dis. 2020;29(8):104941.

23. Horby P, Lim WS, Emberson JR, Mafham M, Bell JL, Linsell L, et al. Dexamethasone in hospitalized patients with Covid-19 — preliminary report. N Engl J Med. 2020 July 17. [Epub ahead of print].

24. Kim SY, Jin W, Sood A, Montgomery DW, Grant OC, Fuster MM, et al Characterization of heparin and severe acute respiratory syndrome-related coronavirus 2 (SARS-CoV-2) spike glycoprotein binding interactions. Antiviral Res. 2020 Sep;181:104873. 